\title{
Mini-Review: Human Microbiome and Rheumatic Diseases
}

\author{
Meltem Vural ${ }^{1 *}$, Benoit Gilbert ${ }^{2}$, Işıl Üstün ${ }^{1}$, Sibel Caglar ${ }^{1}$ and Axel Finckh ${ }^{2}$ \\ 1 Physical Medicine and Rehabilitation Clinic, University of Health Sciences, Bakırkoy Dr. Sadi Konuk Training Hospital, \\ Istanbul, Turkey, ${ }^{2}$ Rheumatology Division, Department of Medicine, Geneva University Hospital (HUG), Geneva, Switzerland
}

Rheumatoid arthritis and spondyloarthropathy are the most common inflammatory rheumatic diseases. As the human microbiome is involved in the immune homeostasis, it has the potential to be a key factor in the development of autoimmune diseases and rheumatic diseases. In this article, we review the role of various human microbiota on the pathogenesis of rheumatic diseases, focusing on spondylarthritis and rheumatoid arthritis.

Keywords: microbiome, rheumatic diseases, etiopathogenesis, spondylarthritis, rheumatoid arthritis

\section{OPEN ACCESS}

Edited by:

Venkatakrishna Rao Jala,

University of Louisville, United States

Reviewed by:

Gyanendra Prakash Dubey,

Institut Pasteur, France

Karl M. Thompson,

Howard University, United States

*Correspondence:

Meltem Vural

meltem.vural@sbu.edu.tr

Specialty section:

This article was submitted to Microbiome in Health and Disease,

a section of the journal

Frontiers in Cellular

and Infection Microbiology

Received: 12 August 2019

Accepted: 14 October 2020

Published: 10 November 2020

Citation:

Vural M, Gilbert B, Üstün I, Caglar S and Finckh A (2020) Mini-Review:

Human Microbiome and

Rheumatic Diseases.

Front. Cell. Infect. Microbiol. 10:491160.

doi: 10.3389/fcimb.2020.491160

\section{INTRODUCTION}

Evidence from animal and human translational research suggests an important function of the microbiome in modulating the host's immunity (Longman and Littman, 2015). Although disruptions in local microbiome composition (dysbiosis) can result in a systemic inflammatory response (Littman and Pamer, 2011; Manasson and Scher, 2015; Scher et al., 2016), the development of an inflammatory disease would require a disturbance of the complex network of interactions between the microbiome, genetic factors and the environment (Kim et al., 2016; PicchiantiDiamanti et al., 2018).

Rheumatic musculoskeletal diseases (RMDs) are the second cause of years lived with disability worldwide (Vos et al., 2015). Rheumatoid Arthritis (RA), is the most prevalent chronic autoimmune disease. It leads to peripheral joint destruction and extra-articular manifestations, usually sparring distal interphalangeal and spinal joints (Majithia and Geraci, 2007). RA incidence rate is still rising (Safiri et al., 2019), and prevalence approximates $0.5 \%$ to $1 \%$ in the European and North American population (Cooper and Stroehla, 2003). Diagnosis is mainly clinical, but identification of auto-antibodies, such as rheumatoid factor or anti-citrullinated-peptides antibodies (ACPA) has a high positive predictive value (Majithia and Geraci, 2007). Unlike RA, spondylarthritis (SpA) refers to a group of clinically and genetically related disorders for which no specific autoantibody has been identified, which explains why the SpA are not considered an autoimmune disease, but classified as an "immune-mediated inflammatory diseases". SpA includes ankylosing spondylitis (AS), psoriatic arthritis (PsA), juvenile SpA, reactive arthritis (ReA), and inflammatory bowel disease (IBD)-related arthritis (Asquith et al., 2014). SpA is considered to have a more heterogeneous clinical spectrum, usually involving spinal joints, in association with peripheral manifestations, distinct from RA symptomatology.

Recent advances in sequencing technologies have greatly improved systematic and comprehensive investigations of the human microbiome (Costello et al., 2015a). DNA-based taxonomy allows

Abbreviations: AS, ankylosing spondylitis; FMT, fecal microbiota transplantation; IBD, inflammatory bowel disease; PsA, psoriatic arthritis; RA, rheumatoid arthritis; ReA, reactive arthritis; SpA, spondylarthritis. 
identification at the species level, while microbial metagenomics and metatranscriptomics respectively inform about functional capacity and actively transcribed genes in the microbiome (Goodrich et al., 2014; Sharpton, 2014; Hiergeist et al., 2015; Coit and Sawalha, 2016). The evolutionary process of coadaptation, as well as the direct and indirect crosstalk between the intestinal microbiome and the host immune system is now increasingly appreciated (Scher et al., 2016; Caminer et al., 2017). Thanks to the use of these novel microbiologic techniques, the human microbiome has increasingly been recognized as a potential key factor in the development of autoimmune diseases (Manasson and Scher, 2015; Scher et al., 2016) and RMDs.

Herein, we review the role of the microbiome in specific human rheumatic diseases focusing on spondylarthritis and rheumatoid arthritis.

\section{HUMAN MICROBIOME IN SPONDYLARTHRITIS}

With a relatively larger participation of genetics in the disease etiopathogenesis, spondylarthritis also remarkably depends on the role of pro-inflammatory Th17 cells and its signature cytokine, IL-17 (Mielants et al., 1995; Scher et al., 2016). Hence, intestinal microbiota, gut inflammation and SpA are considered to have an epidemiological link, as supported by the demonstrated role of infectious diarrhea in the pathogenesis of $\operatorname{ReA}$, and the association between IBD and AS and PsA (Mielants et al., 1995; Arvikar and Fisher, 2011; Li W.-Q. et al., 2013). The Human Leukocyte Antigen B27 (HLA B27) is a MHC Class I molecule. HLA B27 association with SpA is the most relevant $\mathrm{MHC}$ association in human diseases. There are several hypothesis for the role of HLA B27 molecule in SpA: 1) arthritogenic peptide recognized by HLA B27, 2) HLA-B27 protein misfolding resulting in intracellular stress and 3) innate immune recognition of aberrant HLA-B27 (Brown, 2014). The binding of HLA-B27 homodimers to KIR3DL2-positive cells can stimulate IL-17 production, and CD4 T cells positive for KIR3DL2 in the synovial fluid of patients with spondylarthritis demonstrate increased IL-17 secretion, IL-23 receptor expression and production of inflammatory cytokins (Chen L. et al., 2016).

Animal studies have described spontaneous inflammatory SpA-like disease in transgenic rats expressing HLA-B27, which have a phenotype characterized by sacroiliitis, peripheral arthritis, psoriasiform skin inflammation and colitis (Hammer et al., 1990; Taurog et al., 1994). The disease predisposition associated with this allele has been linked to HLA-B27-mediated alterations in the intestinal microbiome by a combination of HLA-B27 misfolding and activation of intestinal and circulating Th17 cells, in an IL-23-dependent manner (DeLay et al., 2009; Rosenbaum and Davey, 2011; Glatigny et al., 2012). Data from other animal models have also indicated the role of gut bacteria in SpA inflammation, such as spontaneous development of progressive enthesitis and ankylosis triggered by commensals in the ANKENT mouse model (Šinkorová et al., 2008).
ANKENT mice having HLA B27 spontaneously develop a progressive ankylosing enthesopathy of ankle and tarsal joints (Weinreich et al., 1995). SKG is a mouse strain that develops chronic autoimmune arthritis similar to RA (Sakaguchi et al., 2003). Also the development of SpA-like disease after a systemic injection of $\beta$-glucan through downstream production of IL-23dependent IL-17/IL-2270 in the SKG mouse model reinforces this issue (Ruutu et al., 2012; Sherlock et al., 2012). A link between IL23R polymorphisms and susceptibility to psoriasis, PsA and AS has also been suggested (Liu et al., 2008).

Hence, a disrupted gut environment is hypothesized to be associated with dysregulated immune response and/or altered dendritic cell function in spondyloarthropathy (Stoll, 2015). There is substantial evidence regarding the important role of the microbiota in SpA through multiple overlapping mechanisms, including alteration of intestinal permeability (possibly determined by genetic factors such as HLA-B27 expression), decreased production of anti-inflammatory metabolic products, activation of intestinal immunity, and migration of microbial products to the peripheral joints with subsequent local immune activation (Rosenbaum and Davey, 2011; Lin et al., 2014; Stoll, 2015; Picchianti-Diamanti et al., 2018).

In a study with 9 AS patients and 9 healthy controls, terminal ileum biopsy specimens were generated using 16S ribosomal RNA gene sequences and analysis techniques in SpA patients, gut microbial analyses reported higher abundance of Lachnospiraceae, Ruminococcaceae, Rikenellaceae, Porphyromonadaceae, and Bacteroidaceae, and lower abundance of Veillonellaceae and Prevotellaceae compared to healthy controls (Costello et al., 2015b). Decreased numbers of Firmicutes, major phyla of gut commensals, particularly Faecalibacterium prausnitzii and Clostridium leptum species were also reported in SpA and IBD patients and considered an important link between SpA and gut inflammation (Gill et al., 2015).

\section{Ankylosing Spondylitis}

A direct link between systemic inflammation and gut dysfunction in humans has been clearly identified only in AS, including several alterations of the ileum architecture, disruption of the basal membrane with compromised epithelial cell permeability, hyperplasia of goblet cells and activation of Paneth cells, producing high levels of adenosine monophosphate and proinflammatory cytokines, such as IL23 (Ciccia et al., 2010; Ciccia et al., 2012; Ciccia et al., 2016, p. 23; Picchianti-Diamanti et al., 2018).

The microbial community of terminal ileal biopsies from AS patients and healthy controls demonstrated the presence of an intestinal dysbiosis, with a more diverse terminal ileum microbiota signature in AS patients compared to healthy controls (Costello et al., 2015b). The dysbiosis in the AS gut was indicated to be driven by higher abundance of five families of bacteria Lachnospiraceae, Ruminococcaceae, Rikenellaceae, Porphyromonadaceae and Bacteroidaceae and a decrease in abundance of two families of Veilonellaceae, without an overall alteration in microbial load or an overgrowth or dominance of a 
specific microbe (Costello et al., 2015b). A higher prevalence of sulfate-reducing bacteria was also reported in patients with AS. The sulfate-reducing bacteria can reduce inorganic sulfate to hydrogen sulfide. They are hydrogen scavengers. Higher prevalence of these bacteria may demonstrate gut inflammation (Stebbings, 2002). In children with enthesitis-related arthritis (according to the International League of Associations for Rheumatology criteria, juvenile idiopathic arthritis subtype), the relative abundance of $F$. prausnitzii was reported to be significantly lower as compared to healthy controls. This finding has also been reported in patients with IBD (Thorkildsen et al., 2013; Stoll et al., 2014).

\section{Psoriatic Arthritis}

The overexpression of IL9 and the production of Th9, Th17, and Th22 responses produce subclinical gut inflammation in PSA patients (Picchianti-Diamanti et al., 2018). Furthermore, gutderived Th9 cells have the ability to migrate and enter synovial tissues, before initiating a positive autocrine loop and local inflammation created via IL9 and IL9 receptor expression by gut-activated Paneth cells (Ciccia et al., 2016).

Analysis of the gut microbiota with high-throughput $16 \mathrm{~S}$ rRNA pyrosequencing method in a cohort of PsA and psoriasis patients demonstrated a decrease in taxonomic diversity due to lower abundances of several taxa compared to the microbiota of healthy controls (Scher et al., 2015), including decreased Coprococcus in both psoriasis and PsA patients, while significantly lower levels of Akkermansia and Ruminococcus only in the PsA group (Scher et al., 2015; Scher et al., 2016). This decline in Ruminococcus and Akkermansia specific to PsA has been interpreted as a chronological loss of diversity that may potentially associate with the natural history of disease (Scher et al., 2015; Scher et al., 2016). Interestingly, a similar reduction of Ruminococcaceae family and Akkermansia genus has also been reported in patients with IBD (Willing et al., 2010; Scher et al., 2016). In addition, the decreased abundance of Akkermansia in PsA contrasts to that of juvenile SpA, emphasizing the likelihood of distinct microbes in the etiology of these diseases (Gill et al., 2015). A hallmark of intestinal dysbiosis in PsA patients is considered to be the loss of commensals responsible for mucus degradation and small and medium chain fatty acid production. This situation suggests a relation between microbiome perturbations, mucosal integrity and the dissemination of systemic inflammation (Gill et al., 2015; Coit and Sawalha, 2016; Scher et al., 2016).

Data from recent $16 \mathrm{~S}$ rRNA sequencing studies have also indicated significant differences in cutaneous microbiota between psoriasis patients and controls, and between psoriatic lesions and control skin samples, with greater abundance of Firmicutes and lesser abundance of phylum Actinobacteria, Staphylococci, and Propionibacteria in cases and in affected skin (Gao et al., 2008; Fahlén et al., 2012; Costello et al., 2015a). Large shifts of normal phyla of the human skin and reduction of Propionibacteria may be the cause or result of drastic changes in diseased skin. It still remains unknown if it is a potential biomarker to predict who eventually develop psoriatic arthritis (Coit and Sawalha, 2016).

\section{Juvenile SpA}

Patients with a specific form of juvenile SpA, classified as enthesitis-related arthritis (ERA), were reported to exhibit decreased abundance of C. leptum, similar to AS patients (Stebbings, 2002; Stoll et al., 2014). Furthermore, a decrease in F. prausnitzii (another member of the Clostridales family) and in cellular immune response to Salmonella typhimurium were also reported in patients with juvenile SpA compared to healthy controls (Singh et al., 2011; Gill et al., 2015).

\section{HUMAN MICROBIOME IN RHEUMATOID ARTHRITIS (RA)}

The etiopathogenesis of RA is partially understood and believed to result from a multi-step process. Various environmental factors would insidiously initiate a pathological activation of the immune system in genetically susceptible individuals (Klareskog et al., 2006). This aberrant immune activation would result in asymptomatic presence of autoantibodies, such as rheumatoid factor or anti-citrullinated-peptide antibodies (ACPA) (Klareskog et al., 2006). A subsequent paucisymptomatic or "pre-clinical" phase usually precedes the clinically apparent evolution toward classifiable RA (Klareskog et al., 2006).

This "immune onset" of the disease is thought to be initiated in extra-articular tissues before localizing in the synovium (Holers et al., 2018). Hence, the mucosal origins hypotheses of RA (Li S et al., 2013) suspect a role of microbiota in dysregulating immune response. Proposed mechanisms (Li S et al., 2013) linking microbes to autoimmunity include: - 1) the microbial generation of neo-autoantigens (such as citrullinated peptides) targeted by the immune system; - 2) a loss of tolerance of the immune system against normal self-antigens via molecular mimicry [e.g. rheumatic fever (Cunningham, 2000)]; - 3) a bystander activation of the immune system via a non-specific activation of auto-reactive $\mathrm{T}$ cells by microbes or microbial super-antigens.

\section{The Oral Microbiota and RA}

Periodontitis is clearly associated with established RA (Potikuri et al., 2012; Scher et al., 2012; Eriksson et al., 2019), but the causal relationship has only been studied in a very limited number of longitudinal studies. Still, participants who had periodontal disease and missing teeth had a higher risk of subsequent incident RA (Demmer et al., 2011).

Interestingly, within population genetically at-risk for RA, ACPA-positives individuals have higher prevalence and severity of periodontitis than ACPA-negative subjects (Loutan et al., 2019). The latter suggests a causal role for periodontitis in the development of systemic auto-immunity preceding RA onset. In animal models, periodontitis primed by Porphyromonas gingivalis transplantation triggers seropositive arthritis after 4 to 8 months, with systemic inflammation and bone erosions (Courbon et al., 2019). P gingivalis has the ability to citrullinate the host's peptides and produce neo-autoantigens. This 
observation initially led to the hypothesis that this microbe could be associated with the etiopathogenesis of RA (Li S et al., 2013). Oral Aggregatibacter actinomycetemcomitans (Aa) was also later shown to induce in vivo hypercitrullination patterns, comparable to those observed in the joints of RA patients (Konig et al., 2016). Moreover at least $47 \%$ of patients with established RA were immune for $A a$, versus only $11 \%$ in healthy controls (Konig et al., 2016). However, current evidence does not allow to point out a given bacterial taxa as the unique responsible for oral dysbiosis in RA development. Instead, several inflammatory-associated species, for instance within the Prevotella or Leptotrichia genus (Table 1), associate with RA and periodontitis (Scher et al., 2012; Chen et al., 2018). Overall, this oral dysbiosis tends to attenuate after adequate antirheumatic treatment (Zhang et al., 2015), suggesting that periodontitis might trigger autoimmunity in a subset of patients, but does not necessarily perpetuates the autoimmune process.

\section{The Gut Microbiota and RA}

Early research between the 1970's and 2000's already suggested quantitative changes of specific bacterial species in DMARDnaive RA patients, including Clostridium perfringens (Olhagen and Månsson, 1968), Bacteroides, Prevotella and Porphyromonas genera (Eerola et al., 1994; Toivanen et al., 2002; Vaahtovuo et al., 2008). After 2010, several studies using 16S RNA/DNA sequencing technology demonstrated an increased relative abundance of Prevotella copri in pre-RA or in early RA patients compared to healthy controls (Scher et al., 2013; Maeda et al., 2016; Alpizar-Rodriguez et al., 2019). Interestingly, this relative expansion of $P$. copri was rarely present in established and chronically treated RA patients or in psoriatic arthritis patients (Scher et al., 2013).

In an animal model of arthritis, transplantation of a human microbiota from RA patients dominated by Prevotella resulted in severe arthritis, which was not the case when healthy-controlderived microbiota was transplanted (Maeda et al., 2016). The authors have thus postulated that $P$. copri might carry an epitope conferring cross-reactivity to arthritis-related autoantigens. Interestingly, Pianta and al. later isolated a peptide presented by monocytes from a chronic RA patient, peptide belonging to P. copri (protein Pc-p27) (Pianta et al., 2017a). Antibody response to this protein or to P. copri was detected in $32 \%$ of new onset RA patients, while rarely found in other forms of arthritis or in healthy controls (Pianta et al., 2017b). Remarkably, a sequence homology exists between two self-proteins targeted by the immune response in RA (Wang et al., 2017) and epitopes from Prevotella species (Pianta et al., 2017a). The latter is suggestive for molecular mimicry and provides a possible mechanism linking intestinal dysbiosis with RA development. However, P. copri is certainly not the only intestinal bacteria associated with the pathogenesis of RA. Other microbes, such as Collinsella aerofaciens, have also been involved, and proved to worsen arthritis in mice models (Chen J. et al., 2016). Moreover, in established RA patients, dysbiosis with different species have also been described (Zhang et al., 2015; Chen J. et al., 2016). Furthermore, species such as Prevotella histicola were even shown to exert a protective effect in mice models
(Marietta et al., 2016). Future studies will thus have to better establish the role of intestinal dysbiosis in the development of autoimmunity and the relative contribution of the different bacterial species. Ultimately, we will have to demonstrate that targeting intestinal dysbiosis in individuals at high risk for RA can prevent the onset of the disease.

\section{FUTURE DIRECTIONS}

Research on the role of microbiota in RMDs has been hampered by the fuzziness of the disease phenotypes studied. The microbiome has been shown to differ between patients with early disease, prior to immunosuppressive therapy, and patients with established and chronically treated disease (Zhang et al., 2015). Moreover, current 16 S profiling typically provides only the relative abundance of various species, thus delivering a proportional result, which may be susceptible to biases by extraneous factors, such as bowel habits (Vandeputte et al., 2017). In addition, the IBD literature has suggested a role of the intestinal virome in IBD-specific dysbiosis (Norman et al., 2015) and underlined colitogenic properties of secretory IgAcoated human bacteria (Palm et al., 2014), which may also be relevant in the pathogenesis of RMDs (Stoll, 2015; Scher et al., 2016). The characterization of IgA-coated bacteria in RA population has never been performed.

Overall, there is a need for more quantitative analysis of the microbiome and in-depth longitudinal metagenome-wide association studies addressing the functional capacity of the host microbiota. This will help answer questions that require rigorous exploration such as the directionality (cause or consequence of autoimmune process) of the dysbiotic process. The timing of microbial perturbation in the clinical course of the disease remains unclear. The contribution of communities in specific body niches (i.e. the periodontal and lung and the skin in PsA) and the potential utility of the microbiome in pharmacokinetics of immunosuppressive therapy are also of interest (Coit and Sawalha, 2016; Scher et al., 2016). Future microbiome research in RMDs needs to focus more on strain level identification of bacteria and overcome limitations of 16Sbased analyses, which might not be precise enough. For example, Palm et al. could demonstrate that bacterial isolates from different IBD patients that were taxonomically assigned as the same species via $16 \mathrm{~S}$ sequencing often displayed differential IgAcoating patterns, corresponding to genetically distinct strains and very different pathological capabilities (Palm et al., 2014).

A therapeutic window of opportunity for anti-rheumatic therapy exists in the early phases of RMDs (Finckh et al., 2006), and it is widely believed that an even earlier intervention, prior to the development of arthritis, could be curative (Finckh and Deane, 2014). In humans, at least six different randomized controlled trials are testing the efficacy of preventive medicinal approaches (Finckh and Deane, 2014). Nonetheless, people at high risk for RMDs appear to prefer non-medicinal interventions, such as dietary interventions, stress reduction or physical exercise (Novotny et al., 2013). Therefore, studies are exploring the efficacy of modifying risk factors of 
TABLE 1 | Disease specific microbiota alterations at the species level and associated relevant biological findings.

\begin{tabular}{|c|c|c|}
\hline Disease & Microbiota alteration & $\begin{array}{l}\text { Relevant associated biological } \\
\text { findings }\end{array}$ \\
\hline
\end{tabular}

\begin{tabular}{ll}
\hline Rheumatoid & Oral microbiota \\
arthritis & -Overall diversity preserved, but different taxa repartition in RA \\
& (Mikuls et al., 2018). In particular, Prevotella species and Leptotrichia \\
& species almost absent in healthy controls (Scher et al., 2012; Chen \\
& et al., 2018),and depletion of Haemophilus spp in RA patients \\
& (Zhang et al., 2015; Corrêa et al., 2019). \\
& -Infection with Porphyromonas gingivalis or Aggregatibacter \\
& actinomycetemcomitans (Aa) (Konig et al., 2016) more frequent in \\
& RA patients.
\end{tabular}

\section{Gut microbiota}

-Increase of Prevotella species, in particular Prevotella copri, in early RA and preclinical stages of RA (Scher et al., 2013).

- Eggerthella genus (Zhang et al., 2015; Chen J et al., 2016), and Collinsella aerofaciens was also more abundant in some RA patients (Chen J et al., 2016).

Ankylosing spondylitis

Psoriatic arthritis

Juvenile spondylarthritis

\section{Gut microbiota}

-Higher abundance of five families of bacteria Lachnospiraceae, Ruminococcaceae, Rikenellaceae, Porphyromonadaceae and Bacteroidaceae, and lower abundance of two families of Veilonellaceae (Costello et al., 2015b) and Prevotellaceae. -A higher prevalence of sulfate-reducing bacteria was also reported in patients with AS.
-Analysis of gingival crevicular fluid from patients with periodontitis revealed extensive protein citrullination with same patterns as observed in RA joints (Konig et al., 2016)

-Incubation of neutrophils with $A a$, but not with $P$. gingivalis, reproduced this hypercitrullination (Konig et al., 2016) - Periodontitis status correlates with anti-citrullinated-peptides antibodies (Corrêa et al., 2019).

- Serological immunity against $P$ copr is specific for RA patients (Pianta et al., 2017b).

- Prevotella species have potentially cross-reactive antigens (Pianta et al., 2017a).

-Patient-derived Collinsella

aerofaciens (Chen J et al., 2016), as well as Prevotella species aggravate arthritis in mice models.

-A higher prevalence of sulfatereducing bacteria, also known as hydrogen scavengers. These bacteria can reduce inorganic sulphate to hydrogen sulfide. The higher prevalence of these bacteria may indicate gut inflammation (Stebbings, 2002).

- The decreased abundance of Ruminococcus and Akkermansia specific to PsA has been evaluated as a chronological loss of diversity that may potentially associate with the natural history of disease (Scher et al., 2015). -The decrease of Propionibacteria may be the cause or result of changes in diseased skin. It still remains unknown if it is a potential biomarker to predict who finally improve PSA (Coit and Sawalha, 2016).

-In children with enthesitis-related arthritis (juvenile idiopathic arthritis subtype), the relative abundance of F. prausnitzii was presented lower as compared to healthy controls (Stoll et al., 2014).
Future directions?

-Unravel associations between smoking, periodontitis, oral dysbiosis

and rheumatoid arthritis onset. -Homogenize sampling methodology across the various oral sites (saliva, crevicular fluid, dental plaque, etc.).
-Longitudinal follow-up of at-risk population to allow proper causal inference.

- Identification of targeted approaches to shape gut microbiota (probiotics, FMT, functional foods, etc.).

-Modifiye risk factors including nutritional habits (fiber-enriched cereal bars or dietary interventions).

- To investigate the effectiveness of fecal transplantation and its long-term results.

- Further studies in AS patient's microbiota needs to focus more on strain level identification of bacteria and plans to study on limitations of 16Sbased analysis.

-Homogenize sampling methodology across the skin both psoriasis and PsA patients.

-Future preventive or treatment strategies could be including microbiota-targeted interventions, such as dietary interventions, probiotics or FMT.

-Planning of further and larger studies on microbiota analysis of children with ERA.

-Education of patients and parents about probiotics and nutritional habits.
RMDs, including nutritional habits (Sparks et al., 2014), change in oral microbiome by treating periodontal disease (University Hospital, Toulouse, 2018) or by administering probiotics (Alipour et al., 2014; Wang et al., 2016). For instance, a double blinded placebo-controlled trial showed that daily supplementation of RA patients with Lactobacillus casei 01 improved disease activity after 8 weeks (Vaghef-Mehrabany et al., 2014). Nevertheless, other studies with different Lactobacillus strains were inconclusive (de los
Angeles Pineda et al., 2011). Overall, a meta-analysis published in 2017 concluded that even though probiotics decreased inflammatory cytokines, clinical effect in established RA was still unclear (Mohammed et al., 2017). Whether these conclusions apply to at-risk population, as a preventive measure prior to disease occurrence, has not been studied yet.

Another potentially attractive intervention for intestinal dysbiosis is fecal microbiota transplantation (FMT), which is 
defined as the administration of fecal micro-organisms from a healthy donor into the digestive tract of a recipient patient. FMT is a successful treatment for Clostridium difficile infections (Surawicz et al., 2013; Cammarota et al., 2017), and is currently tested in immune mediated diseases, such as ulcerative colitis (Moayyedi et al., 2015; Rossen et al., 2015a; Paramsothy et al., 2017). It is indeed considered to be a safe procedure (Rossen et al., 2015b; Cohen and Maharshak, 2017), even in debilitated or immunosuppressed patients (Agrawal et al., 2016; Friedman-Korn et al., 2018) although long-term side effects are largely unknown. No study has yet established the feasibility and safety of FMT in RMDs. However, studies with FMT are ongoing in psoriatic arthritis patients (Kragsnaes et al., 2018) and RA patients. Functional foods, such as fiber-enriched cereal bars (Häger et al., 2019), or dietary interventions, could also be considered as future tools for shaping intestinal flora, even though solid evidence in this field is still lacking.

\section{CONCLUSION}

The direct and indirect interplay between the microbiome and the host immune responses is now increasingly appreciated, as more human microbiome studies become available and the mucosal immunity is better understood (Scher et al., 2016; Caminer et al., 2017). High-throughput sequencing has become a valuable tool, with the potential to offer a new biologic basis for classification and clues for pathogenesis in RMDs (Gill et al., 2015; Coit and Sawalha, 2016; Scher et al., 2016). So far, various dysbiosis have been identified in the microbiota of patients with specific RMDs compared to

\section{REFERENCES}

Agrawal, M., Aroniadis, O. C., Brandt, L. J., Kelly, C., Freeman, S., Surawicz, C., et al. (2016). The Long-term Efficacy and Safety of Fecal Microbiota Transplant for Recurrent, Severe, and Complicated Clostridium difficile Infection in 146 Elderly Individuals. J. Clin. Gastroenterol. 50 (5), 403-407. doi: 10.1097/ MCG.0000000000000410

Alipour, B., Homayouni-Rad, A., Vaghef-Mehrabany, E., Sharif, S. K., VaghefMehrabany, L., Asghari-Jafarabadi, M., et al. (2014). Effects of Lactobacillus casei supplementation on disease activity and inflammatory cytokines in rheumatoid arthritis patients: a randomized double-blind clinical trial. Int. J. Rheum. Dis. 17 (5), 519-527. doi: 10.1111/1756-185X.12333

Alpizar-Rodriguez, D., Lesker, T. R., Gronow, A., Gilbert, B., Raemy, E., Lamacchia, C., et al. (2019). Prevotella copri in individuals at risk for rheumatoid arthritis. Ann. Rheum. Dis. 78 (5), 590-593. doi: 10.1136/annrheumdis-2018-214514

Arvikar, S. L., and Fisher, M. C. (2011). Inflammatory bowel disease associated arthropathy. Curr. Rev. Musculoskelet Med. 4 (3), 123-131. doi: 10.1007/ s12178-011-9085-8

Asquith, M., Elewaut, D., Lin, P., and Rosenbaum, J. T. (2014). The role of the gut and microbes in the pathogenesis of spondyloarthritis. Best Pract. Res. Clin. Rheumatol. 28 (5), 687-702. doi: 10.1016/j.berh.2014.10.018

Brown, A. (2014). "Genetics of axial spondyloarthritis," in Rheumatology, sixth edition. Ed. M. C. Hochberg. Available at: https://doi.org/10.1016/B978-0-32309138-1.00116-9.

Caminer, A. C., Haberman, R., and Scher, J. U. (2017). Human microbiome, infections, and rheumatic disease. Clin. Rheumatol. 36 (12), 2645-2653. doi: 10.1007/s10067-017-3875-3 controls (Alpizar-Rodriguez et al., Stebbings, 2002; Scher et al., 2013; Stoll et al., 2014; Costello et al., 2015b; Gill et al., 2015; Scher et al., 2015; Maeda et al., 2016). Furthermore, gut microbiota from diseased patients has been shown to trigger or exacerbate disease phenotypes in RMDs mouse models (Šinkorová et al., 2008; Ruutu et al., 2012; Sherlock et al., 2012; Maeda et al., 2016). Etiopathogenic hypotheses generally involve a microbiota related activation of intestinal Th9 (Ciccia et al., 2016), Th17 cells (DeLay et al., 2009; Rosenbaum and Davey, 2011; Glatigny et al., 2012), or molecular mimicry with mucosal bacteria (Pianta et al., 2017a; Pianta et al., 2017b; Wang et al., 2017). However, many more questions remain to be addressed before a causal relationship of the human microbiome in the pathogenesis of rheumatic diseases is definitely established. The specific role of the microbiota in the development of RMDs and exact immunological mechanisms need to be better understood (Gill et al., 2015; Scher et al., 2016; Coit and Sawalha, 2016), while methodology needs to be harmonized and updated. If the findings of this line of research hold true, the translation into clinical practice would result in novel preventive or curative strategies, which could include microbiota-targeted interventions, such as dietary modifications, functional foods, probiotics, or FMT.

\section{AUTHOR CONTRIBUTIONS}

MV, BG, IÜ, SC, and AF contributed to the design and implementation of the review and to the writing of the manuscript. All authors contributed to the article and approved the submitted version.

Cammarota, G., Ianiro, G., Tilg, H., Rajilic-Stojanovic, M., Kump, P., Satokari, R., et al. (2017). European consensus conference on faecal microbiota transplantation in clinical practice. Gut 66 (4), 569-580. doi: 10.1136/gutjnl2016-313017

Chen, B., Zhao, Y., Li, S., Yang, L., Wang, H., Wang, T., et al. (2018). Variations in oral microbiome profiles in rheumatoid arthritis and osteoarthritis with potential biomarkers for arthritis screening. Sci. Rep. 8 (1), 17126. doi: 10.1038/s41598-018-35473-6

Chen, J., Wright, K., Davis, J. M., Jeraldo, P., Marietta, E. V., Murray, J., et al. (2016). An expansion of rare lineage intestinal microbes characterizes rheumatoid arthritis. Genome Med. 8 (1), 43. doi: 10.1186/s13073-016-0299-7

Chen, L., Ridley, A., Hammitzsch, A., Al-Mossawi, M. H., Bunting, H, Georgiadis, D., et al. (2016). Silencing or inhibition of endoplasmic reticulum aminopeptidase 1 (ERAP1) suppresses free heavy chain expression and Th17 responses in ankylosing spondylitis. Ann. Rheum. Dis. 75 (5), 916-923. doi: 10.1136/annrheumdis-2014-206996

Ciccia, F., Bombardieri, M., Rizzo, A., Principato, A., Giardina, A. R., Raiata, F., et al. (2010). Over-expression of paneth cell-derived anti-microbial peptides in the gut of patients with ankylosing spondylitis and subclinical intestinal inflammation. Rheumatology 49 (11), 2076-2083. doi: 10.1093/ rheumatology/keq239

Ciccia, F., Accardo-Palumbo, A., Alessandro, R., Rizzo, A., Principe, S., Peralta, S., et al. (2012). Interleukin-22 and interleukin-22-producing NKp44+ natural killer cells in subclinical gut inflammation in ankylosing spondylitis. Arthritis Rheum. 64 (6), 1869-1878. doi: 10.1002/art.34355

Ciccia, F., Guggino, G., Ferrante, A., Raimondo, S., Bignone, R., Rodolico, V., et al. (2016). Interleukin-9 Overexpression and Th9 Polarization Characterize the 
Inflamed Gut, the Synovial Tissue, and the Peripheral Blood of Patients With Psoriatic Arthritis: IL-9 IN PsA. Arthritis Rheumatol. 68 (8), 1922-1931. doi: 10.1002/art.39649

Cohen, N. A., and Maharshak, N. (2017). Novel Indications for Fecal Microbial Transplantation: Update and Review of the Literature. Dig. Dis. Sci. 62 (5), 1131-1145. doi: 10.1007/s10620-017-4535-9

Coit, P., and Sawalha, A. H. (2016). The human microbiome in rheumatic autoimmune diseases: A comprehensive review. Clin. Immunol. 170, 70-79. doi: 10.1016/j.clim.2016.07.026

Cooper, G. S., and Stroehla, B. C. (2003). The epidemiology of autoimmune diseases. Autoimmun. Rev. 2 (3), 119-125. doi: 10.1016/S1568-9972(03)00006-5

Corrêa, J. D., Fernandes, G. R., Calderaro, D. C., Souza Mendonça, S. M., Silva, J. M., Albiero, M. L., et al (2019). Oral microbial dysbiosis linked to worsened periodontal condition in rheumatoid arthritis patients. Sci Rep. 9 (1), 1-10. doi: 10.1038/s41598-019-44674-6

Costello, M.-E., Robinson, P. C., Benham, H., and Brown, M. A. (2015a). The intestinal microbiome in human disease and how it relates to arthritis and spondyloarthritis. Best Pract. Res. Clin. Rheumatol. 29 (2), 202-212. doi: 10.1016/j.berh.2015.08.001

Costello, M.-E., Ciccia, F., Willner, D., Warrington, N., Robinson, P. C., Gardiner, B., et al. (2015b). Brief Report: Intestinal Dysbiosis in Ankylosing Spondylitis: Gut Microbiome and AS-Related Genes. Arthritis Rheumatol. 67 (3), 686-691. doi: 10.1002/art.38967

Courbon, G., Rinaudo-Gaujous, M., Blasco-Baque, V., Auger, I., Caire, R., Mijola, L., et al. (2019). Porphyromonas gingivalis experimentally induces periodontis and an anti-CCP2-associated arthritis in the rat. Ann. Rheum. Dis. 78 (5), 594-599. doi: 10.1136/annrheumdis-2018-213697

Cunningham, M. W. (2000). Pathogenesis of group A streptococcal infections. Clin. Microbiol. Rev. 13 (3), 470-511. doi: 10.1128/cmr.13.3.470-511.2000

de los Angeles Pineda, M., Thompson, S. F., Summers, K., de Leon, F., Pope, J., and Reid, G. (2011). A randomized, double-blinded, placebo-controlled pilot study of probiotics in active rheumatoid arthritis. Med. Sci. Monit. 17 (6), CR347CR354. doi: 10.12659/MSM.881808

DeLay, M. L., Turner, M. J., Klenk, E. I., Smith, J. A., Sowders, D. P., and Colbert, R. A. (2009). HLA-B27 misfolding and the unfolded protein response augment interleukin-23 production and are associated with Th17 activation in transgenic rats. Arthritis Rheumatol. 60 (9), 2633-2643. doi: 10.1002/art.24763

Demmer, R. T., Molitor, J. A., Jacobs, D. R., and Michalowicz, B. S. (2011). Periodontal disease, tooth loss and incident rheumatoid arthritis: results from the First National Health and Nutrition Examination Survey and its epidemiological follow-up study. J. Clin. Periodontol. 38 (11), 998-1006. doi: 10.1111/j.1600-051X.2011.01776.x

Eerola, E., Möttönen, T., Hannonen, P., Luukkainen, R., Kantola, I., Vuori K., et al. (1994). Intestinal Flora in Early Rheumatoid Arthritis. Rheumatol. (Oxford) 33 (11), 1030-1038. doi: 10.1093/rheumatology/33.11.1030

Eriksson, K., Fei, G., Lundmark, A., Benchimol, D., Lee, L., Hu, Y. O. O., et al. (2019). Periodontal Health and Oral Microbiota in Patients with Rheumatoid Arthritis. J. Clin. Med. 8 (5) doi: 10.3390/jcm8050630

Fahlén, A., Engstrand, L., Baker, B. S., Powles, A., and Fry, L. (2012). Comparison of bacterial microbiota in skin biopsies from normal and psoriatic skin. Arch. Dermatol. Res. 304 (1), 15-22. doi: 10.1007/s00403-011-1189-x

Finckh, A., and Deane, K. D. (2014). Prevention of Rheumatic Diseases: Strategies, Caveats, and Future Directions. Rheum. Dis. Clinics North America 40 (4), 771-785. doi: 10.1016/j.rdc.2014.07.010

Finckh, A., Liang, M. H., van Herckenrode, C. M., and de Pablo, P. (2006). Longterm impact of early treatment on radiographic progression in rheumatoid arthritis: A meta-analysis. Arthritis Care Res. 55 (6), 864-872. doi: 10.1002/ art.22353

Friedman-Korn, T., Livovsky, D. M., Maharshak, N., Aviv Cohen, N., Paz, K., Bar-Gil Shitrit, A., et al. (2018). Fecal Transplantation for Treatment of Clostridium Difficile Infection in Elderly and Debilitated Patients. Digest. Dis. Sci. 63 (1), 198-203. doi: 10.1007/s10620-017-4833-2

Gao, Z., Tseng, C., Strober, B. E., Pei, Z., and Blaser, M. J. (2008). Substantial Alterations of the Cutaneous Bacterial Biota in Psoriatic Lesions. Ahmed N, ed. PloS One 3 (7), e2719. doi: 10.1371/journal.pone.0002719

Gill, T., Asquith, M., Rosenbaum, J. T., and Colbert, R. A. (2015). The intestinal microbiome in spondyloarthritis. Curr. Opin. Rheumatol. 27 (4), 319-325. doi: 10.1097/BOR.0000000000000187
Glatigny, S., Fert, I., Blaton, M. A., Lories, R. J., Araujo, L. M., Chiocchia, G, et al. (2012). Proinflammatory Th17 cells are expanded and induced by dendritic cells in spondylarthritis-prone HLA-B27-transgenic rats. Arthritis Rheum. 64 (1), 110-120. doi: 10.1002/art.33321

Goodrich, J. K., Di Rienzi, S. C., Poole, A. C., Koren, O, Walters, W. A., Caporaso, J. G., et al. (2014). Conducting a Microbiome Study. Cell 158 (2), 250-262. doi: $10.1016 /$ j.cell.2014.06.037

Häger, J., Bang, H., Hagen, M., Frech, M., Träger, P., Sokolova, M. V., et al. (2019). The Role of Dietary Fiber in Rheumatoid Arthritis Patients: A Feasibility Study. Nutrients 11 (10), 2392. doi: 10.3390/nu11102392

Hammer, R. E., Maika, S. D., Richardson, J. A., Tang, J.-P., and Taurog, J. D. (1990). Spontaneous inflammatory disease in transgenic rats expressing HLAB27 and human $32 \mathrm{~m}$ : An animal model of HLA-B27-associated human disorders. Cell 63 (5), 1099-1112. doi: 10.1016/0092-8674(90)90512-D

Hiergeist, A., Gläsner, J., Reischl, U., and Gessner, A. (2015). Analyses of Intestinal Microbiota: Culture versus Sequencing: Figure 1. ILAR J. 56 (2), 228-240. doi: 10.1093/ilar/ilv017

Holers, V. M., Demoruelle, M. K., Kuhn, K. A., Buckner, J. H., Robinson, W. H., Okamoto, Y., et al. (2018). Rheumatoid arthritis and the mucosal origins hypothesis: protection turns to destruction. Nat. Rev. Rheumatol. 14 (9), $542-$ 557. doi: 10.1038/s41584-018-0070-0

Kim, K. S., Hong, S.-W., Han, D., Yi, J., Jung, J., Yang, B-G., et al. (2016). Dietary antigens limit mucosal immunity by inducing regulatory $\mathrm{T}$ cells in the small intestine. Science 351 (6275), 858-863. doi: 10.1126/science.aac5560

Klareskog, L., Stolt, P., Lundberg, K., Källberg, H., Bengtsson, C., Grunewald, J., et al. (2006). A new model for an etiology of rheumatoid arthritis: Smoking may trigger HLA-DR (shared epitope)-restricted immune reactions to autoantigens modified by citrullination. Arthritis Rheum. 54 (1), 38-46. doi: 10.1002/art.21575

Konig, M. F., Abusleme, L., Reinholdt, J., Palmer, R. J., Teles, R. P., Sampson, K., et al. (2016). Aggregatibacter actinomycetemcomitans-induced hypercitrullination links periodontal infection to autoimmunity in rheumatoid arthritis. Sci. Trans. Med. 8 (369), 369ra176-369ra176. doi: 10.1126/scitranslmed. aaj1921

Kragsnaes, M. S., Kjeldsen, J., Horn, H. C., Munk, H. L., Pedersen, F. M., Holt, H. M., et al. (2018). Efficacy and safety of faecal microbiota transplantation in patients with psoriatic arthritis: protocol for a 6-month, double-blind, randomised, placebo-controlled trial. BMJ Open 8 (4), e019231. doi: 10.1136/ bmjopen-2017-019231

Li, S., Yu, Y., Yue, Y., Zhang, Z., and Su, K. (2013). Microbial Infection and Rheumatoid Arthritis. J. Clin. Cell Immunol. 4 (6). doi: 10.4172/21559899.1000174

Li, W.-Q., Han, J.-L., Chan, A. T., and Qureshi, A. A. (2013). Psoriasis, psoriatic arthritis and increased risk of incident Crohn's disease in US women. Ann. Rheum. Dis. 72 (7), 1200-1205. doi: 10.1136/annrheumdis-2012-202143

Lin, P., Bach, M., Asquith, M., Lee, A. Y., Akileswaran, L., Stauffer, P., et al. (2014). HLA-B27 and Human 32 -Microglobulin Affect the Gut Microbiota of Transgenic Rats. Bereswill S, ed. PloS One 9 (8), e105684. doi: 10.1371/ journal.pone. 0105684

Littman, D. R., and Pamer, E. G. (2011). Role of the Commensal Microbiota in Normal and Pathogenic Host Immune Responses. Cell Host Microbe 10 (4), 311-323. doi: 10.1016/j.chom.2011.10.004

Liu, Y., Helms, C., Liao, W., Zaba, L. C., Duan, S., Gardner, J., et al. (2008). A Genome-Wide Association Study of Psoriasis and Psoriatic Arthritis Identifies New Disease Loci. Leal SM, ed. PloS Genet. 4 (4), e1000041. doi: 10.1371/ journal.pgen.1000041

Longman, R. S., and Littman, D. R. (2015). The Functional Impact of the Intestinal Microbiome on Mucosal Immunity and Systemic Autoimmunity. Curr. Opin. Rheumatol. 27 (4), 381-387. doi: 10.1097/BOR.0000000000000190

Loutan, L., Alpizar-Rodriguez, D., Courvoisier, D. S., Finckh, A., Mombelli, A., and Giannopoulou, C. (2019). Periodontal status correlates with anticitrullinated protein antibodies in first-degree relatives of individuals with rheumatoid arthritis. J. Clin. Periodontol. 46 (7), 690-698. doi: 10.1111/ jcpe. 13117

Maeda, Y., Kurakawa, T., Umemoto, E., Motooka, D., Ito, Y., Gotoh, K., et al. (2016). Dysbiosis Contributes to Arthritis Development via Activation of Autoreactive T Cells in the Intestine. Arthritis Rheumatol. 68 (11), 26462661. doi: 10.1002/art.39783 
Majithia, V., and Geraci, S. A. (2007). Rheumatoid Arthritis: Diagnosis and Management. Am. J. Med. 120 (11), 936-939. doi: 10.1016/ j.amjmed.2007.04.005

Manasson, J., and Scher, J. U. (2015). Spondyloarthritis and the Microbiome: New Insights From an Ancient Hypothesis. Curr. Rheumatol. Rep. 17 (2):10. doi: 10.1007/s11926-014-0487-7

Marietta, E. V., Murray, J. A., Luckey, D. H., Jeraldo, P. R., Lamba, A., Patel, R., et al. (2016). Suppression of Inflammatory Arthritis by Human Gut-Derived Prevotella histicola in Humanized Mice. Arthritis Rheumatol. 68 (12), 28782888. doi: 10.1002/art.39785

Mielants, H., Veys, E. M., De Vos, M., Cuvelier, C., Goemaere, S., De Clercq, L., et al. (1995). The evolution of spondyloarthropathies in relation to gut histology. I. Clinical aspects. J. Rheumatol. 22 (12), 2266-2272.

Mikuls, T. R., Walker, C., Qiu, F., Yu, F., Thiele, G. M., Alfant, B., et al. (2018). The subgingival microbiome in patients with established rheumatoid arthritis. Rheumatology (Oxford). 57 (7), 1162-1172. doi: 10.1093/rheumatology/key052

Moayyedi, P., Surette, M. G., Kim, P. T., Libertucci, J., Wolfe, M., Onischi, C., et al. (2015). Fecal Microbiota Transplantation Induces Remission in Patients With Active Ulcerative Colitis in a Randomized Controlled Trial. Gastroenterology 149 (1), 102-109 e6. doi: 10.1053/j.gastro.2015.04.001

Mohammed, A. T., Khattab, M., Ahmed, A. M., Turk, T., Sakr, N. M., Khalil, A., et al. (2017). The therapeutic effect of probiotics on rheumatoid arthritis: a systematic review and meta-analysis of randomized control trials. Clin. Rheumatol. 36 (12), 2697-2707. doi: 10.1007/s10067-017-3814-3

Norman, J. M., Handley, S. A., Baldridge, M. T., Droit, L., Liu, C. Y., Keller, B. C., et al. (2015). Disease-Specific Alterations in the Enteric Virome in Inflammatory Bowel Disease. Cell 160 (3), 447-460. doi: 10.1016/ j.cell.2015.01.002

Novotny, F., Haeny, S., Hudelson, P., Escher, M., and Finckh, A. (2013). Primary prevention of rheumatoid arthritis: a qualitative study in a high-risk population. Joint Bone Spine 80 (6), 673-674. doi: 10.1016/j.jbspin.2013.05.005

Olhagen, B., and Månsson, I. (1968). Intestinal Clostridium Perfringens in Rheumatoid Arthritis and Other Collagen Diseases1. Acta Med. Scand. 184 (1-6), 395-402. doi: 10.1111/j.0954-6820.1968.tb02478.x

Palm, N. W., de Zoete, M. R., TW, C., Barry, N. A., Stefanowski, J., Hao, L., et al. (2014). Immunoglobulin A Coating Identifies Colitogenic Bacteria in Inflammatory Bowel Disease. Cell 158 (5), 1000-1010. doi: 10.1016/ j.cell.2014.08.006

Paramsothy, S., Kamm, M. A., Kaakoush, N. O., Walsh, A. J., van den Bogaerde, J., Samuel, D., et al. (2017). Multidonor intensive faecal microbiota transplantation for active ulcerative colitis: a randomised placebo-controlled trial. Lancet 389 (10075), 1218-1228. doi: 10.1016/S0140-6736(17)30182-4

Pianta, A., Arvikar, S. L., Strle, K., Drouin, E. E., Wang, Q., Costello, C. E., et al. (2017a). Two rheumatoid arthritis-specific autoantigens correlate microbial immunity with autoimmune responses in joints. J. Clin. Invest. 127 (8), 29462956. doi: 10.1172/JCI93450

Pianta, A., Arvikar, S., Strle, K., Drouin, E. E., Wang, Q., Costello, C. E., et al. (2017b). Evidence of the Immune Relevance of Prevotella copri, a Gut Microbe, in Patients With Rheumatoid Arthritis: Immune Relevance of Prevotella Copri in Patients With RA. Arthritis Rheumatol. 69 (5), 964-975. doi: 10.1002/ art.40003

Picchianti-Diamanti, A., Rosado, M. M., and D'Amelio, R. (2018). Infectious Agents and Inflammation: The Role of Microbiota in Autoimmune Arthritis. Front. Microbiol. 8, 2696. doi: 10.3389/fmicb.2017.02696

Potikuri, D., Dannana, K. C., Kanchinadam, S., Agrawal, S., Kancharla, A., Rajasekhar, L., et al. (2012). Periodontal disease is significantly higher in non-smoking treatment-naive rheumatoid arthritis patients: results from a case-control study. Ann. Rheum. Dis. 71 (9), 1541-1544. doi: 10.1136/ annrheumdis-2011-200380

Rosenbaum, J. T., and Davey, M. P. (2011). Time for a gut check: Evidence for the hypothesis that HLA-B27 predisposes to ankylosing spondylitis by altering the microbiome. Arthritis Rheum. 63 (11), 3195-3198. doi: 10.1002/art.30558

Rossen, N. G., Fuentes, S., van der Spek, M. J., Tijssen, J. G., Hartman, J. H., Duflou, A., et al. (2015a). Findings From a Randomized Controlled Trial of Fecal Transplantation for Patients With Ulcerative Colitis. Gastroenterology 149 (1), 110-118.e4. doi: 10.1053/j.gastro.2015.03.045

Rossen, N. G., MacDonald, J. K., de Vries, E. M., D’Haens, G. R., de Vos, W. M., Zoetendal, E. G., et al. (2015b). Fecal microbiota transplantation as novel therapy in gastroenterology: A systematic review. World J. Gastroenterol. 21 (17), 5359-5371. doi: 10.3748/wjg.v21.i17.5359

Ruutu, M., Thomas, G., Steck, R., Degli-Esposti, M. A., Zinkernagel, M. S., Alexander, K., et al. (2012). B-glucan triggers spondylarthritis and Crohn's disease-like ileitis in SKG mice. Arthritis Rheum. 64 (7), 2211-2222. doi: 10.1002/art.34423

Safiri, S., Kolahi, A. A., Hoy, D., Smith, E., Bettampadi, D., Mansournia, M. A., et al. (2019). Global, regional and national burden of rheumatoid arthritis 1990-2017: a systematic analysis of the Global Burden of Disease study 2017. Ann. Rheum. Dis. 78 (11), 1463-1471. doi: 10.1136/annrheumdis-2019-215920

Sakaguchi, S., Takahashi, T., Hata, H., Nomura, T., and Sakaguchi, N. (2003). SKG mice, a new genetic model of rheumatoid arthritis. Arthritis Res. Ther. 5 (Suppl 3):10. doi: 10.1186/ar811

Scher, J. U., Ubeda, C., Equinda, M., Khanin, R., Buischi, Y., Viale, A., et al. (2012). Periodontal disease and the oral microbiota in new-onset rheumatoid arthritis. Arthritis Rheum. 64 (10), 3083-3094. doi: 10.1002/art.34539

Scher, J. U., Sczesnak, A., Longman, R. S., Segata, N., Ubeda, C., Craig Bielski, C., et al. (2013). Expansion of intestinal Prevotella copri correlates with enhanced susceptibility to arthritis. eLife 2 doi: 10.7554/eLife.01202

Scher, J. U., Ubeda, C., Artacho, A., Attur, M., Isaac, S., and Reddy, S. M. (2015). Decreased Bacterial Diversity Characterizes the Altered Gut Microbiota in Patients With Psoriatic Arthritis, Resembling Dysbiosis in Inflammatory Bowel Disease: Gut Microbiota in PsA. Arthritis Rheumatol. 67 (1), 128-139. doi: 10.1002/art.38892

Scher, J. U., Littman, D. R., and Abramson, S. B. (2016). Review: Microbiome in Inflammatory Arthritis and Human Rheumatic Diseases: MICROBIOME IN RHEUMATIC DISEASES. Arthritis Rheumatol. 68 (1), 35-45. doi: 10.1002/ art.39259

Sharpton, T. J. (2014). An introduction to the analysis of shotgun metagenomic data. Front. Plant Sci. 5, 209. doi: 10.3389/fpls.2014.00209

Sherlock, J. P., Joyce-Shaikh, B., Turner, S. P., Chao, C-C., Sathe, M., Grein, J., et al. (2012). IL-23 induces spondyloarthropathy by acting on ROR- $\gamma \mathrm{t}+\mathrm{CD} 3+\mathrm{CD} 4-$ CD8- entheseal resident T cells. Nat. Med. 18 (7), 1069-1076. doi: 10.1038/ nm.2817

Singh, Y. P., Singh, A. K., Aggarwal, A., and Misra, R. (2011). Evidence of Cellular Immune Response to Outer Membrane Protein of Salmonella typhimurium in Patients with Enthesitis-related Arthritis Subtype of Juvenile Idiopathic Arthritis. J. Rheumatol. 38 (1), 161-166. doi: 10.3899/jrheum.100542

Šinkorová, Z., Čapková, J., Niederlová, J., Štěpánková, R., and Šinkora, J. (2008). Commensal intestinal bacterial strains trigger ankylosing enthesopathy of the ankle in inbred B10.BR (H-2k) male mice. Hum. Immunol. 69 (12), 845-850. doi: 10.1016/j.humimm.2008.08.296

Sparks, J. A., Iversen, M. D., Miller Kroouze, R., Mahmoud, T. G., Triedman, N. A., Kalia, S. S., et al. (2014). Personalized Risk Estimator for Rheumatoid Arthritis (PRE-RA) Family Study: Rationale and design for a randomized controlled trial evaluating rheumatoid arthritis risk education to first-degree relatives. Contemp. Clin. Trials 39 (1), 145-157. doi: 10.1016/j.cct.2014.08.007

Stebbings, S. (2002). Comparison of the faecal microflora of patients with ankylosing spondylitis and controls using molecular methods of analysis. Rheumatology 41 (12), 1395-1401. doi: 10.1093/rheumatology/41.12.1395

Stoll, M. L., Kumar, R., Morrow, C. D., Lefkowitz, E. J., Cui, X., Genin, A., et al. (2014). Altered microbiota associated with abnormal humoral immune responses to commensal organisms in enthesitis-related arthritis. Arthritis Res. Ther. 16 (6), 486. doi: 10.1186/s13075-014-0486-0

Stoll, M. L. (2015). Gut microbes, immunity, and spondyloarthritis. Clin. Immunol. 159 (2), 134-142. doi: 10.1016/j.clim.2015.05.001

Surawicz, C. M., Brandt, L. J., Binion, D. G., Ananthakrishnan, A. N., Curry, S. R., Gilligan, P. H., et al. (2013). Guidelines for diagnosis, treatment, and prevention of Clostridium difficile infections. Am. J. Gastroenterol. 108 (4), 478-498; quiz 499. doi: 10.1038/ajg.2013.4

Taurog, J. D., Richardson, J. A., Croft, J. T., Simmons, W. A., Zhou, M., FernándezSueiro, J. L., et al. (1994). The germfree state prevents development of gut and joint inflammatory disease in HLA-B27 transgenic rats. J. Exp. Med. 180 (6), 2359-2364. doi: 10.1084/jem.180.6.2359

Thorkildsen, L. T., Nwosu, F. C., Avershina, E., Ricanek, P., Perminow, G., Brackmann, S., et al. (2013). Dominant Fecal Microbiota in Newly Diagnosed Untreated Inflammatory Bowel Disease Patients. Gastroenterol. Res. Pract. 2013, 1-13. doi: 10.1155/2013/636785 
Toivanen, P., Vartiainen, S., Jalava, J., Luukkainen, R., Möttönen, T., Eerola, E., et al. (2002). Intestinal anaerobic bacteria in early rheumatoid arthritis (RA). Arthritis Res. Ther. 4 (1), 5. doi: 10.1186/ar492

University Hospital, Toulouse (2018). Efficacy of Therapeutic Management of Periodontitis on the Clinical Manifestations of Rheumatoid Arthritis: The Randomized, Controlled ESPERA Trial. clinicaltrials.gov. Available at: https:// clinicaltrials.gov/ct2/show/NCT02779179 (Accessed July 21, 2020).

Vaahtovuo, J., Munukka, E., Korkeamäki, M., Luukkainen, R., and Toivanen, P. (2008). Fecal microbiota in early rheumatoid arthritis. J. Rheumatol. 35 (8), 1500-1505.

Vaghef-Mehrabany, E., Alipour, B., Homayouni-Rad, A., Sharif, S.-K., AsghariJafarabadi, M., and Zavvari, S. (2014). Probiotic supplementation improves inflammatory status in patients with rheumatoid arthritis. Nutrition 30 (4), 430-435. doi: 10.1016/j.nut.2013.09.007

Vandeputte, D., Kathagen, G., D’hoe, K., Vieira-Silva, S., Valles-Colomer, M., Sabino, J., et al. (2017). Quantitative microbiome profiling links gut community variation to microbial load. Nature 551 (7681), 507-511. doi: $10.1038 /$ nature 24460

Vos, T., Barber, R. M., Bell, B., Bertozzi-Villa, A., Biryukov, S., Bolliger, I., et al. (2015). Global, regional, and national incidence, prevalence, and years lived with disability for 301 acute and chronic diseases and injuries in 188 countries, 1990-2013: a systematic analysis for the Global Burden of Disease Study 2013. Lancet 386 (9995), 743-800. doi: 10.1016/S0140-6736(15)60692-4

Wang, P., Tao, J.-H., and Pan, H.-F. (2016). Probiotic bacteria: a viable adjuvant therapy for relieving symptoms of rheumatoid arthritis. Inflammopharmacology 24 (5), 189-196. doi: 10.1007/s10787-016-0277-0
Wang, Q., Drouin, E. E., Yao, C., Zhang, J., Huang, Y., Leon, D. R., et al. (2017). Immunogenic HLA-DR-Presented Self-Peptides Identified Directly from Clinical Samples of Synovial Tissue, Synovial Fluid, or Peripheral Blood in Patients with Rheumatoid Arthritis or Lyme Arthritis. J. Proteome Res. 16 (1), 122-136. doi: 10.1021/acs.jproteome.6b00386

Weinreich, S., Eulderink, F., Capkova, J., Pla, M., Gaede, K., Heesemann, J., et al. (1995). HLA-B27 as a relative risk factor in ankylosing enthesopathy in transgenic mice. Hum. Immunol. 42 (2), 103-115. doi: 10.1016/0198-8859(94)00034-N

Willing, B. P., Dicksved, J., Halfvarson, J., Andersson, A. F., Lucio, M., Zheng, Z., et al. (2010). A Pyrosequencing Study in Twins Shows That Gastrointestinal Microbial Profiles Vary With Inflammatory Bowel Disease Phenotypes. Gastroenterology 139 (6), 1844-1854.e1. doi: 10.1053/j.gastro.2010.08.049

Zhang, X., Zhang, D., Jia, H., Feng, Q., Wang, D., Liang, D., et al. (2015). The oral and gut microbiomes are perturbed in rheumatoid arthritis and partly normalized after treatment. Nat. Med. 21 (8), 895-905. doi: 10.1038/nm.3914

Conflict of Interest: The authors declare that the research was conducted in the absence of any commercial or financial relationships that could be construed as a potential conflict of interest.

Copyright (c) 2020 Vural, Gilbert, Üstün, Caglar and Finckh. This is an open-access article distributed under the terms of the Creative Commons Attribution License (CC BY). The use, distribution or reproduction in other forums is permitted, provided the original author(s) and the copyright owner(s) are credited and that the original publication in this journal is cited, in accordance with accepted academic practice. No use, distribution or reproduction is permitted which does not comply with these terms. 